This item was submitted to Loughborough's Research Repository by the author.

Items in Figshare are protected by copyright, with all rights reserved, unless otherwise indicated.

\title{
Self-similar correlations in a barrier billiard
}

PLEASE CITE THE PUBLISHED VERSION

\section{LICENCE}

CC BY-NC-ND 4.0

\section{REPOSITORY RECORD}

Chapman, J.R., and Andrew H. Osbaldestin. 2019. "Self-similar Correlations in a Barrier Billiard". figshare. https://hdl.handle.net/2134/465. 


\title{
Self-similar correlations in a barrier billiard
}

\author{
J. R. Chapman and A. H. Osbaldestin* \\ Department of Mathematical Sciences, Loughborough University \\ Loughborough, LE11 3TU, UK
}

August 19, 2002

\begin{abstract}
We give a renormalization analysis of the self-similarity of autocorrelation functions in symmetric barrier billiards for golden mean trajectories. For the special case of a half-barrier we present a rigorous calculation of the asymptotic height of the main peaks in the autocorrelation function. Fundamental to this work is a detailed analysis of a functional recurrence equation which has previously been used in the analysis of fluctuations in the Harper equation and of correlations in strange non-chaotic attractors and in quantum two-level systems.
\end{abstract}

PACS: 05.45.-a, 64.60.Ak

Keywords: Barrier billiards, renormalization

\section{Introduction}

Barrier billiards form a class of dynamical systems that appear to be neither regular nor chaotic. Investigating the nature of the dynamics, Wiersig [17] has recently provided numerical evidence that for such billiards typical phase space functions exhibit a spectrum with a singular continuous component. Central to Wiersig's analysis is a skew-product evolution equation for the sign of a phase-space variable. (We shall give a complete derivation in section 2 below.) Previously Riley [16] had shown that these same skew-products do indeed typically display singular continuous spectra. (See [19] and references therein for results on the weak-mixing nature of such billiards using interval-exchange transformations.) Of interest to use here is that, in addition, Wiersig provides evidence that autocorrelation functions neither decay to zero nor return to 1. Moreover, for the system parameters and initial conditions chosen, the autocorrelation function shows a self-similar form. (See figure 3 below for an example.) This form of autocorrelation function is identical to that witnessed in the strange non-chaotic attractors present in quasiperiodically driven systems [15] — further examples of skew-product systems. In that context, for the case of forcing at golden mean frequency, the self-similarity is explained in [5] by means of a renormalization analysis, a central ingredient being the functional recurrence

$$
Q_{n}(x)=Q_{n-1}(-\omega x) Q_{n-2}\left(\omega^{2} x+\omega\right)
$$

where $\omega=(\sqrt{5}-1) / 2$ is the golden mean. The self-similarity arises as a result of the initial conditions for this recurrence (which are dictated by the system parameters) leading to periodic behaviour. In [11] we have given a complete analysis of the relevant periodic orbits of (1.1), namely piecewise-constant functions taking the values \pm 1 , thereby putting the work in [5] on a firmer footing.

Ketoja and Satija [8] had previously shown how periodic orbits of this same recurrence also help explain self-similar fluctuations of eigenfunctions in the Harper equation. (The manner in which strange non-chaotic attractors arise in consideration of the Harper equation was first described in [1] and is further discussed

*corresponding author, email: A.H.Osbaldestin@lboro.ac.uk, fax: +44 1509 223969, phone: +44 1509223189 
in [9].) In [14] we have put the numerical work in [8] on a firm rigorous foundation. In this context the form of the iterates of (1.1) is much more complicated, no longer being simple piecewise-constant functions. In [8] Ketoja and Satija also discover a universal renormalization strange set - the orchid - associated with a generalised Harper equation. In [13] we show how indeed recurrence (1.1) generates such a set. On the subject of the structure of a strange non-chaotic attractor itself, Kuznetsov et al [10] also utilise (1.1). We anticipate that our work on the orchid in [13] will throw some light on the this problem.

Similar phenomena are observed, and explained by renormalization, in the related problem of a quasiperiodically forced two-level quantum system [6]. An additive version of (1.1), which may be thought of as a functional (quantised) version of the classical Fibonacci recursion, is the governing recursion in this case. In [12] we have given a complete description of the periodic orbits of this recursion thereby explaining and generalising the results in [6].

Inspired by the article of Wiersig [17], in this article adapt our work in [11] and [12] to the symmetric barrier billiard problem. We shall study golden mean trajectories and see that, once again, the self-similarity of the autocorrelation function is explained in terms of periodic orbits of (1.1). We shall give a detailed calculation for the special case of the half-barrier, which, in particular, will include a calculation of the asymptotic height of the main peaks in figure 3 .

We note that recursion (1.1) is relevant no matter what the barrier, given that the angle of the trajectory in the billiard is fixed at the golden mean. Of course, in order to understand the precise nature of the "pseudointegrable" dynamics of barrier billiards, it would be desirable to fix a barrier and consider all trajectories instead. This challenging issue is the subject of ongoing research.

The outline of remainder of this article is as follows. In section 2 we describe symmetric barrier billiards and derive (1.1) as a key equation in the understanding of the autocorrelation function for golden mean trajectories. The initial conditions for (1.1), crucial to the consequent dynamics, will be seen to be determined solely by the details of the barrier. In section 3 we review our earlier work $[11,12]$ on periodic orbits of (1.1) and the locations of their discontinuities. In section 4 we give a detailed analysis for the halfbarrier. We shall show that the heights of the main peaks in the autocorrelation function (figure 3) are asymptotically $1-1 / \sqrt{5} \approx 0.55$, occurring at every third Fibonacci number, whilst for other Fibonacci numbers the autocorrelation is zero. This latter statement was a triviality in the quasiperiodically forced two-level quantum system [12], but requires detailed analysis here.

\section{Symmetric barrier billiards}

Closely following Wiersig [17], in this section we give a self-contained account of how the dynamics of a symmetric barrier billiard may be expressed as a simple skew-product system. We then go on to explain how, for golden mean trajectories, the functional recurrence equation (1.1) arises in the analysis of correlations. The initial conditions for the recurrence are crucial, and will be seen to be dependent on the precise form of the barrier.

\subsection{Equations of motion}

The motion of a point unit mass in the square chamber $[0,1] \times[0,1]$ undergoing elastic collisions according to the law that angle of incidence equals angle of reflection may be described by angles $\theta_{x}, \theta_{y}$ whose time evolution is given by

$$
\begin{aligned}
& \theta_{x}(t)=\theta_{x, 0}+\omega_{x} t \quad(\bmod 1) \\
& \theta_{y}(t)=\theta_{y, 0}+\omega_{y} t \quad(\bmod 1),
\end{aligned}
$$


where $\omega_{x}=p_{x} / 2, \omega_{y}=p_{y} / 2$, with $p_{x}, p_{y}$ signed (constant) one-dimensional velocities. (We ignore trajectories that hit corners.) A key quantity is the rotation number

$$
\omega=\frac{\omega_{y}}{\omega_{x}}=\frac{p_{y}}{p_{x}},
$$

which fixes the direction of motion of the mass. If $\omega$ is rational the motion is periodic, whilst if it is irrational we have quasiperiodic motion densely exploring the configuration space with uniform distribution. This system (rectangular billiards) is an integrable Hamiltonian system. (Without loss of generality we have chosen a square billiard, the general rectangle being equivalent by coordinate transformation.)

The original coordinates of the mass are given by $x=f\left(\theta_{x}\right), y=f\left(\theta_{y}\right)$, where $f(\theta)=2 \theta, \theta \in[0,1 / 2]$, and $f(\theta)=2-2 \theta, \theta \in[1 / 2,1]$. This coordinate transformation becomes invertible once a convention is adopted so that, for instance, $\theta \in[0,1 / 2]$ corresponds to motion with positive velocity.

We now consider the rectangular chamber $[-1,1] \times[0,1]$ in which a vertical barrier (perhaps consisting of many pieces) is located centrally as in figure 1. The barrier is described by the "barrier function" $B:[0,1] \rightarrow\{-1,+1\}$ defined so that $B(y)=+1$ if the barrier is present at $y$ and $B(y)=-1$ if the barrier is absent at $y$. The side figure in figure 1 shows the barrier function for the given barrier.
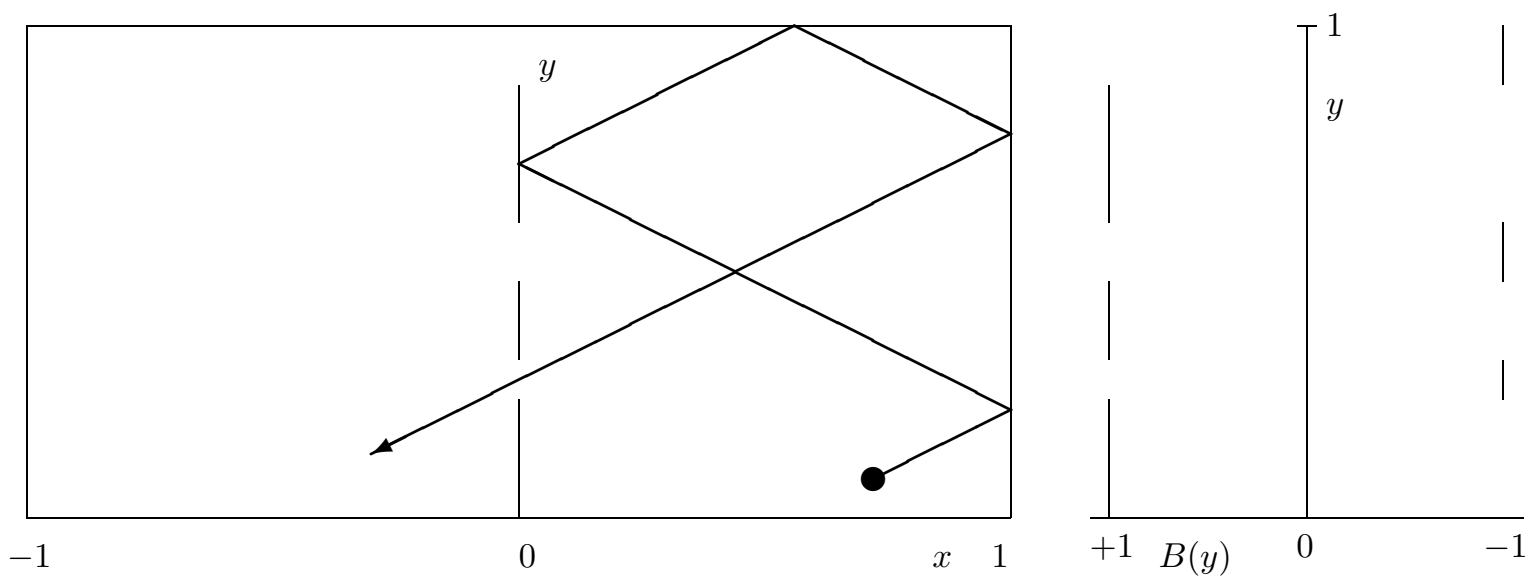

Figure 1: A symmetric barrier billiard and its corresponding barrier function $B(y)$.

We first note that the evolution of $(|x|, y)$ is merely that of rectangular billiards in $[0,1] \times[0,1]$ as described above. (In addition to ignoring trajectories that hit corners we also ignore those that hit barrier ends.) Writing

$$
x(t)=s(t)|x(t)|,
$$

so that $s$ is the sign of $x$ (which we leave undefined whenever $x=0$ ), we will understand this "barrier billiard" system if we understand the evolution of $s$.

Now $s(t)= \pm 1$ and may only change sign at times $t$ such that $x(t)=\theta_{x}(t)=0$. Taking initial condition $x(0)=0$ then $x(t)=0$ if and only if $t=n / \omega_{x}$, where $n \in \mathbb{Z}$. Taking a section $x=0$ (equivalently a stroboscopic sample with frequency $\omega_{x}$ ), for $n \in \mathbb{Z}$ we define

$$
\begin{aligned}
& s_{n}=s\left(n / \omega_{x}^{-}\right) \\
& \theta_{n}=\theta_{y}\left(n / \omega_{x}\right) .
\end{aligned}
$$

Related to $\theta_{n}$ we define $y_{n}=y\left(n / \omega_{x}\right)$, so that $y_{n}=f\left(\theta_{n}\right)$.

The evolution of $\theta_{n}$ is straightforward. From (2.2) we have

$$
\theta_{n+1}=\theta_{n}+\omega_{y} / \omega_{x} \quad(\bmod 1) .
$$

The evolution of $s_{n}$, the sign of $x$ for $t \in\left((n-1) / \omega_{x}, n / \omega_{x}\right)$, is determined by the barrier function $B(y)$. If $y_{n}$ is such that $B\left(y_{n}\right)=+1$ so that the barrier is present then reflection occurs and there is no change of 
sign. On the other hand if $B\left(y_{n}\right)=-1$ so that there is a gap then the mass passes through to the other side of the chamber and thus $s$ changes sign. We thus have

$$
s_{n+1}=s_{n} B\left(y_{n}\right) .
$$

However, we need to express this evolution in terms of the angle $\theta_{n}$. We thus define

$$
\Phi(\theta)=B(f(\theta))=B(y) .
$$

Now it is an important remark that the function $\Phi$ inherits the symmetry of $f$, i.e., $f(1-x)=f(x)$, reflecting the fact that the mass "bounces" from the top of the chamber and "sees" the barrier both on its way upwards and downwards. The barrier function in angle coordinates for the barrier of figure 1 is shown in figure 2 .

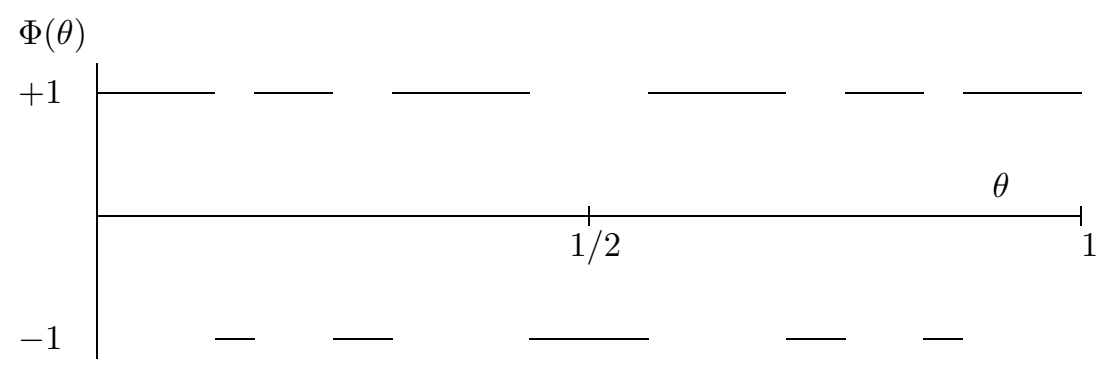

Figure 2: The barrier function $\Phi(\theta)$ for the billiard of figure 1. Note that $\Phi(1-\theta)=\Phi(\theta)$.

In summary, the symmetric barrier billiard may be understood in terms of the skew-product system

$$
\begin{aligned}
& \theta_{n+1}=\theta_{n}+\omega \quad(\bmod 1), \\
& s_{n+1}=s_{n} \Phi\left(\theta_{n}\right) .
\end{aligned}
$$

We remark that this particular skew-product form, crucial for our development here, arises as a consequence of the barrier being symmetrically placed. In his analysis of a circular Andreev billiard, Wiersig [18] uses an asymmetrically placed barrier which leads to a loss of the skew-product form. See also [4] for studies of asymmetric barriers.

Now the system (2.10-2.11) has solution

$$
\begin{aligned}
& \theta_{n}=\theta_{0}+n \omega \quad(\bmod 1), \\
& s_{n}=s_{0} \prod_{k=0}^{n-1} \Phi\left(\theta_{0}+k \omega\right),
\end{aligned}
$$

and for rational rotation number $\omega=p / q$, say with $(p, q)=1$, it is straightforward, using the periodicity of $\theta_{n}$ to verify that $s_{n+q}=F s_{n}$ for all $n$, where the constant $F=\prod_{k=0}^{q-1} \Phi\left(\theta_{k}\right)= \pm 1$, so that $s_{n}$ has period $q$ or $2 q$. However, in the case of irrational rotation number the solution (2.13) does not illuminate the behaviour of the sign $s_{n}$. To this end we turn to an analysis of its autocorrelation function.

\subsection{Autocorrelations}

To understand the behaviour of $s_{n}$ in the case of irrational rotation numbers we look at its autocorrelation function

$$
C(t)=\left\langle s_{n} s_{n+t}\right\rangle=\lim _{N \rightarrow \infty} \frac{1}{N} \sum_{n=1}^{N} s_{n} s_{n+t},
$$

(Note that $\left\langle s_{n}^{2}\right\rangle=1$ since $s_{n}= \pm 1$, and $\left\langle s_{n}\right\rangle=0$ since $\theta_{n}$ is uniformly distributed for irrational $\omega$. Also our average may also be considered to be taken over all initial $s_{0}$ and $\theta_{0}$.) 
As in [5] (and [6], [11], [12]), we have (using the fact that $\theta_{n}$ is uniformly distributed)

$$
\begin{aligned}
C(t) & =\left\langle s_{0} \prod_{k=0}^{n-1} \Phi\left(\theta_{0}+k \omega\right) s_{0} \prod_{k=0}^{n+t-1} \Phi\left(\theta_{0}+k \omega\right)\right\rangle \\
& =\left\langle\prod_{k=n}^{n+t-1} \Phi\left(\theta_{0}+k \omega\right)\right\rangle \\
& =\left\langle\prod_{k=0}^{t-1} \Phi\left(\theta_{n}+k \omega\right)\right\rangle \\
& =\int_{0}^{1} \prod_{k=0}^{t-1} \Phi(\theta+k \omega) d \theta \\
& =\int_{0}^{1} S_{t}(\theta) d \theta
\end{aligned}
$$

where we have defined

$$
S_{t}(\theta)=\prod_{k=0}^{t-1} \Phi(\theta+k \omega), t \geq 1, \quad S_{0}(\theta)=1 .
$$

Figure 3 is a numerical evaluation of the autocorrelation function for the half-barrier which we consider in Section 4 below in the case of golden mean rotation number $\omega$. We shall give a rigorous calculation showing that the magnitude of the main peaks in this figure are asymptotically $1-1 / \sqrt{5} \approx 0.55$, occurring at every third Fibonacci number, and that the autocorrelation is zero for all other Fibonacci numbers.
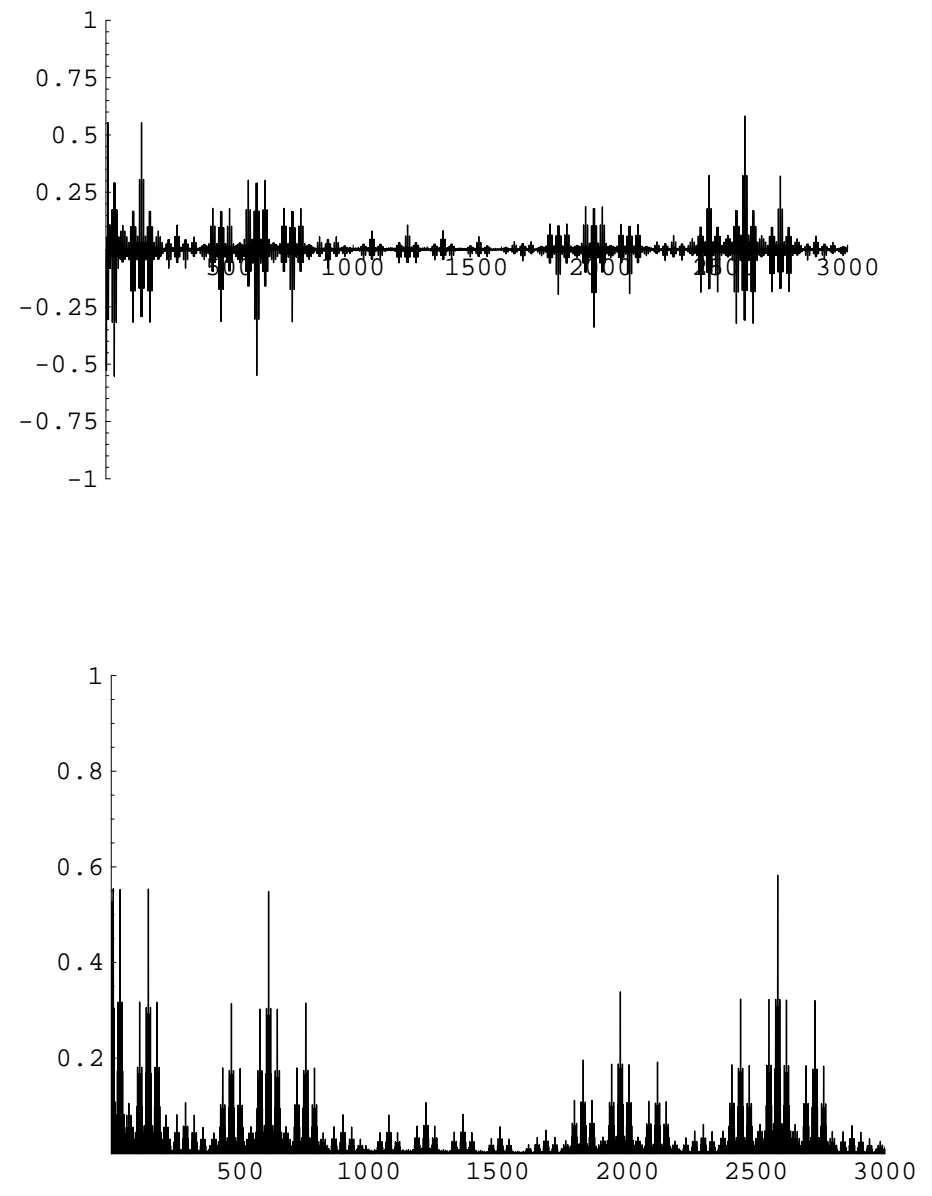

Figure 3: Autocorrelation function $C$ (top), and absolute value $|C|$ (bottom) for the golden mean trajectory in the half-barrier. 


\subsection{Golden mean renormalization}

We concentrate now on the case of golden mean rotation number, so that $\omega=(\sqrt{5}-1) / 2$. We refer to the trajectory of the mass as the "golden mean trajectory." The rational approximants to $\omega$ are now the ratios of successive Fibonacci numbers $\left(F_{n}\right)$ where $F_{0}=0, F_{1}=1$, and $F_{n}=F_{n-1}+F_{n-2}$ for $n \geq 2$. The period-1 functions $S_{t}$ then satisfy a recurrence relation at Fibonacci indices. Indeed we have

$$
\begin{aligned}
S_{F_{n}}(\theta) & =\prod_{k=0}^{F_{n}-1} \Phi\left(\theta_{0}+k \omega\right) \\
& =\prod_{k=0}^{F_{n}-1} \Phi\left(\theta_{0}+k \omega\right) \prod_{k=F_{n-1}}^{F_{n}-1} \Phi\left(\theta_{0}+k \omega\right) \\
& =S_{F_{n-1}}(\theta) \prod_{k=0}^{F_{n}+F_{n-1}-1} \Phi\left(\theta_{n}+F_{n-1} \omega+k \omega\right) \\
& =S_{F_{n-1}}(\theta) \prod_{k=0}^{F_{n-2}-1} \Phi\left(\theta_{n}+F_{n-1} \omega+k \omega\right) \\
& =S_{F_{n-1}}(\theta) S_{F_{n-2}}\left(\theta+F_{n-1} \omega\right)
\end{aligned}
$$

Now we renormalise $S_{F_{n}}$ defining

$$
Q_{n}(x)=S_{F_{n}}\left((-\omega)^{n} x\right),
$$

as a result of which the recurrence (2.17) becomes

$$
Q_{n}(x)=Q_{n-1}(-\omega x) Q_{n-2}\left(\omega^{2} x+\omega\right) .
$$

This recurrence has been the object of attention in a number of our recent works [11]-[14], previously being derived in the manner described in this subsection a variety of contexts ([8], [5], [10]). Of crucial importance is the class of functions it is defined on. This is dictated by the initial conditions, which in this case are easily seen to be

$$
Q_{0}(x)=1, \quad Q_{1}(x)=\Phi(-\omega x) .
$$

We are thus led to consider piecewise-constant functions taking the values \pm 1 . An important observation is that $Q_{1}$ inherits a symmetry from $\Phi$, so that $Q_{1}$ cannot be chosen with arbitrary discontinuity locations.

In terms of the renormalised functions, the autocorrelation function $C(t)$ at Fibonacci times is

$$
C\left(F_{n}\right)=\int_{0}^{1} S_{F_{n}}(\theta) d \theta=\frac{1}{(-\omega)^{-n}} \int_{0}^{(-\omega)^{-n}} Q_{n}(x) d x .
$$

In order to calculate this average we need to consider the global behaviour of the function $Q_{n}$.

\section{Periodic orbits}

In this section we summarise relevant key points from [11, 12] concerning the piecewise-constant periodic orbits of (1.1). These periodic orbits are intimately related to the periodic orbits of a piecewise-linear map of an interval, which in turn are given by the initial conditions to (1.1).

\subsection{Iterated function system and the inverse map $F$}

With $\omega=(\sqrt{5}-1) / 2$, the golden mean, we set

$$
\phi_{1}(x)=-\omega x, \quad \phi_{2}(x)=\omega^{2} x+\omega,
$$

so that we may write equation (1.1) in the form

$$
Q_{n}(x)=Q_{n-1}\left(\phi_{1}(x)\right) Q_{n-2}\left(\phi_{2}(x)\right) .
$$


The iterated function system (IFS) on $\mathbb{R}$ given by $\phi_{1}, \phi_{2}$ has the property that the fundamental interval $I=[-\omega, 1]$ is its fixed point set. Indeed $\phi_{1}(I)=\left[-\omega, \omega^{2}\right], \phi_{2}(I)=\left[\omega^{2}, 1\right]$, so that $\phi_{1}(I) \cup \phi_{2}(I)=I$. The fundamental interval $I$ is the attractor for the IFS: Given any compact subset $K \subset \mathbb{R}$ and any $\varepsilon>0$, there exists $N \in \mathbb{N}$ such that for any $k \geq N$ and any choice $i_{1}, \ldots, i_{k} \in\{1,2\}$ we have $\phi_{i_{1}} \circ \cdots \circ \phi_{i_{k}}(x) \in[-\omega-\varepsilon, 1+\varepsilon]$ for any $x \in K$. This property is important for an understanding of the behaviour of equation (3.2) outside the fundamental interval.

Let $F: I \rightarrow I$ be defined by

$$
F(x)= \begin{cases}\phi_{1}^{-1}(x)=-\omega^{-1} x, & x \in\left[-\omega, \omega^{2}\right] ; \\ \phi_{2}^{-1}(x)=\omega^{-2} x-\omega^{-1}, & x \in\left[\omega^{2}, 1\right],\end{cases}
$$

as drawn in figure 4.

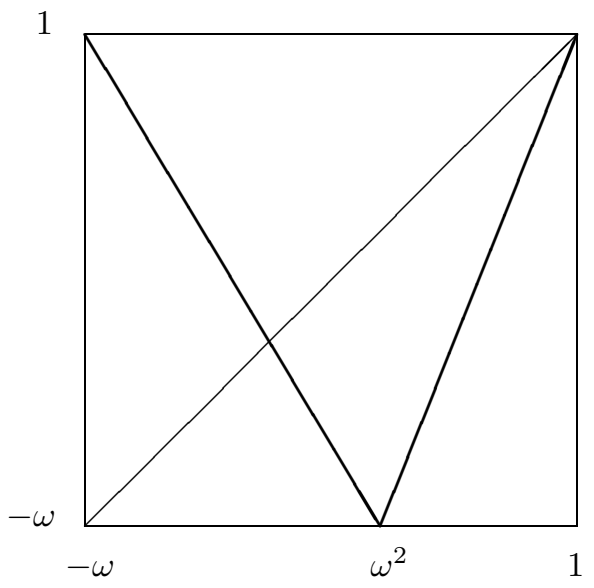

Figure 4: The function $F$.

In [11] it was shown that periodic points of $F$ correspond to discontinuities of the periodic solutions of (3.2) in the fundamental interval. More precisely in [11] we prove the following.

Proposition 1. Let $Q_{n}$ be a piecewise-constant periodic orbit of (3.2) with $Q_{n}(x)= \pm 1$. Then the combined set of discontinuities of all of the elements of the periodic orbit in the fundamental interval consists of a finite collection of periodic orbits of the map F.

To each point $x \in I$ we associate a 'code' defined as the sequence $\left(c_{n}\right)_{n \geq 0}$ in $\{1,2\}^{\mathbb{N}_{0}}$ where

$$
c_{n}= \begin{cases}1, & F^{n}(x) \in\left[-\omega, \omega^{2}\right) ; \\ 2, & F^{n}(x) \in\left(\omega^{2}, 1\right] .\end{cases}
$$

(Our notation here is that $\mathbb{N}_{0}=\mathbb{N} \cup\{0\}=\{0,1,2,3, \ldots\}$.) Since $\omega^{2}$ is not periodic under $F$ we choose to ignore the (countable) set of points whose orbits under $F$ include the point $\omega^{2}$. Each point $x \in I$ corresponds to a unique code and vice versa. Periodic orbits of $F$ correspond to periodic codes in $\{1,2\}^{\mathbb{N}_{0}}$ under the shift $\operatorname{map} \sigma: \sigma\left(c_{0} c_{1} c_{2} \ldots\right)=c_{1} c_{2} \ldots$

A periodic orbit $\left\{y_{0}, y_{1}, \ldots, y_{k-1}\right\}$ of period $k$ of $F$ is given uniquely by a periodic code $\overline{c_{0} c_{1} \ldots c_{k-1}}$, which we henceforth denote as just $c_{0} c_{1} \ldots c_{k-1}$. It is straightforward to calculate the corresponding periodic orbit $y_{0}, y_{1}, \ldots, y_{k-1}$ of $F$, which we may write as $F(x)=\left(-\omega^{-1}\right)^{c_{0}} x-\left(c_{0}-1\right) \omega^{-1}$, for, given a code $c_{0} c_{1} \ldots c_{k-1}$, the solution to $\phi_{c_{k-1}}^{-1} \circ \cdots \circ \phi_{c_{0}}^{-1}\left(y_{0}\right)=y_{0}$, or, equivalently, $\phi_{c_{0}} \circ \cdots \circ \phi_{c_{k-1}}\left(y_{0}\right)=y_{0}$, is readily calculated to be

$$
y_{0}=\frac{-\sum_{j=0}^{k-1}\left(c_{j}-1\right)(-\omega)^{1+\sum_{i=0}^{j-1} c_{i}}}{1-(-\omega)^{\Sigma c_{j}}}
$$

where empty sums are defined to be zero. The other points of the orbit may be calculated by applying this formula with the code $c_{0} c_{1} \ldots c_{k-1}$ cyclically permuted. 
In [11] it was shown that the periodic orbit of (3.2) corresponding to a given code has period dividing the code sum $\sum_{j=0}^{k-1} c_{j}$. Moreover a detailed analysis was given of the periodicity in the presence of multiple periodic orbits of $F$.

\subsection{Reduction of $Q_{n}$ on $\mathbb{R}$ to the fundamental interval}

In what follows we shall need to consider equation (3.2) on the whole of $\mathbb{R}$, and not just in the fundamental interval $I=[-\omega, 1]$. However, the fact that $I$ is the attractor for the IFS leads to the conclusion that the global behaviour of (3.2) is 'driven' by its behaviour in $I$.

From [11] we have the following results.

Lemma 1. Let $Q_{0}, Q_{1}$ be initial conditions for (3.2) on $\mathbb{R}$ and let $\varepsilon>0$ be such that $Q_{0}(x)=Q_{1}(x)=1$ for all $x \in[-\omega-\varepsilon, 1+\varepsilon]$. Then for each $L>1$, there exists $N>0$ (depending only on $L$ ) such that $Q_{n}(x)=1$ for all $x \in[-L, L]$ and all $n>N$.

In other words, if the initial conditions on, and just outside, the fundamental interval are unity, then the value of $Q_{n}$ at all points eventually becomes unity.

The lemma leads to the following propositions.

Proposition 2. Let $Q_{n}$ be a piecewise-constant periodic orbit of (3.2) of period p on $\mathbb{R}$ with $Q_{n}(1+)=Q_{n}(1)$. Then $Q_{n}$ is periodic with period $p$ on the fundamental interval $I$. Conversely, suppose that $Q_{n}$ is periodic with period $p$ on $I$. Then there is a unique extension $\tilde{Q}_{n}$ of $Q_{n}$ to $\mathbb{R}$ such that $\tilde{Q}_{n}$ is periodic with period $p$ on $\mathbb{R}$.

Proposition 3. Let $Q_{0}, Q_{1}$ be piecewise-constant initial conditions for (3.2) on $\mathbb{R}$ with $Q_{0}(1+)=Q_{0}(1)$, $Q_{1}(1+)=Q_{1}(1)$. Suppose $Q_{n}$ is periodic of period $p$ on the fundamental interval $I$. Then the sequence $Q_{n}$ converges to the unique periodic extension $\tilde{Q}_{n}$ given by Proposition 2, i.e., for all integers $r \geq 0$ we have $Q_{r+n p}(x) \rightarrow \tilde{Q}_{r}(x)$ as $n \rightarrow \infty$.

\subsection{Discontinuity locations for periodic orbits}

Let $L_{n}$ denote the set of locations of the discontinuities of $Q_{n}$, then from (3.2) it is clear that

$$
L_{n}=\phi_{1}^{-1}\left(L_{n-1}\right) \cup \phi_{2}^{-1}\left(L_{n-2}\right),
$$

unless there is a cancellation of discontinuities (which may occur if both 1 and 0 are discontinuities). As stated in Subsection 3.2, the global discontinuities of $Q_{n}$ are generated from those in the fundamental interval $I$, and the latter consist of elements of periodic orbits of the map $F$. It is clear from (3.5) that such periodic orbits must be composed of elements of the field $\mathbb{Q}(\omega)=\{a+b \omega: a, b \in \mathbb{Q}\}$. As a consequence, the sets $L_{n}$ consist of elements of $\mathbb{Q}(\omega)$, since the maps $\phi_{1}^{-1}, \phi_{2}^{-1}$ act on $\mathbb{Q}(\omega)$ as

$$
\begin{aligned}
& \phi_{1}^{-1}(a+b \omega)=-(a+b)-a \omega, \\
& \phi_{2}^{-1}(a+b \omega)=2 a+b-1+(a+b-1) \omega .
\end{aligned}
$$

Rather than consider a globally periodic orbit itself, we need to consider an orbit asymptotic to it generated from discontinuity data on the fundamental interval only. By the results of Subsection 3.2 this orbit is eventually periodic and identical to the desired periodic orbit on any bounded subset of $\mathbb{R}$.

With the notation that, for $x \in \mathbb{R},\lceil x\rceil$ denote the ceiling of $x$, namely $\min \{n \in \mathbb{Z}: n \geq x\}$, in [12] we prove the following fundamental result identifying the discontinuity locations.

Proposition 4. The discontinuity location sets $\left(L_{n}\right)$ arising from applying recurrence (3.2) to initial conditions in which $Q_{0}$ has a single discontinuity at $a+b \omega \in I$, and $Q_{1}$ has a single discontinuity at $\phi_{1}^{-1}(a+b \omega)$ are $L_{0}=\{a+b \omega\}$ and for $n \geq 1$

$$
L_{n}=c^{(n)}+d^{(n)} \omega+M_{n}
$$


where

$$
M_{n}=\left\{\lceil i \omega\rceil+i+i \omega: i=l_{n}, \ldots, r_{n}\right\}
$$

with

$$
l_{n}, r_{n}= \begin{cases}-F_{n-1}, F_{n}-1, & n \text { odd } \\ -F_{n}, F_{n-1}-1, & n \text { even }\end{cases}
$$

and where

$$
c^{(n)}=(-1)^{n}\left(F_{n+1} a+F_{n} b\right), \quad d^{(n)}=(-1)^{n}\left(F_{n} a+F_{n-1} b\right) .
$$

\section{The half-barrier}

For the remainder of this article we confine our attention to the particular barrier known as the "half-barrier." In the coordinates of figure 1 , this barrier consists of the segment $[0,1 / 2]$ on the $y$-axis. The corresponding barrier function in angle coordinates is drawn in figure 5. Note the symmetry $\Phi(1-\theta)=\Phi(\theta)$, and recall that $\Phi$ is extended with period one. The half-barrier was studied by Hannay and McCraw [7], as a simple

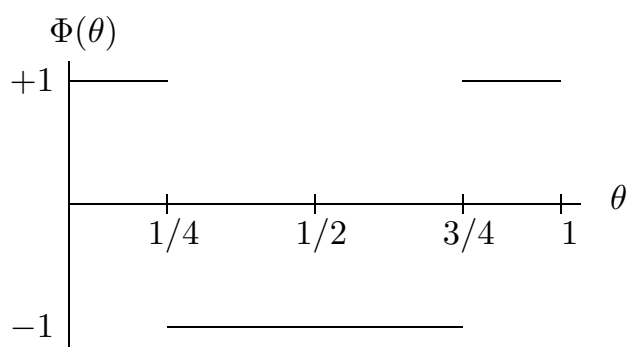

Figure 5: The barrier function $\Phi(\theta)$ for the half-barrier.

example of a pseudo-integrable system. In [17] Wiersig formulates the problem of a barrier consisting of a single piece of arbitrary length and gives numerical results for the half-barrier. Barriers consisting of a single piece have also been studied by others, in particular, Eckhardt et al [3] consider the $60^{\circ}-120^{\circ}$ rhombus billiard which may be considered as a barrier billiard with single barrier of length $2 / 3$. Underpinning these works is the result of Riley [16], that for irrational trajectories almost all such barriers have singular continuous spectrum. See also [19] and references therein for results on the weak-mixing nature of such billiards. In fact an arbitrary barrier function $\Phi$ may be decomposed as a product of barrier functions corresponding to barriers of of this type, the multiplicative structure of (3.2) allowing for separate treatment of the constituent pieces. We shall treat the problem of an arbitrary barrier in a later article, exploiting our work on the universal renormalization strange set arising in a generalised Harper equation [13].

\subsection{Relevant periodic orbits and initial conditions}

According to (2.20) the initial conditions for (3.2) are $Q_{0}(x)=1$, and $Q_{1}(x)=\Phi(-\omega x)$. With $\Phi$ as above the discontinuities of $Q_{1}$ inside the fundamental interval are thus located at $\pm \omega^{-1} / 4= \pm(1 / 4+\omega / 4)$. The orbits of these points under $F$ are

$$
\begin{array}{cccccc}
-1 / 4-\omega / 4 & \mapsto 1 / 2+\omega / 4 & \mapsto 1 / 4-\omega / 4 & -\omega / 4 & \mapsto 1 / 4 & \mapsto-1 / 4-\omega / 4 \\
1 & 2 & 1 & 1 & 1 &
\end{array}
$$

and

$$
\begin{array}{cccccc}
1 / 4+\omega / 4 & \mapsto-1 / 4-\omega / 2 & \mapsto 3 / 4+\omega / 4 & \mapsto 3 / 4 & \mapsto 1 / 2-\omega / 4 & \mapsto-1 / 4-\omega / 2 \\
2 & 1 & 2 & 2 & 1 &
\end{array}
$$

where beneath the points we indicate the code. We see that $-1 / 4-\omega / 4$ is on the periodic orbit $P^{1}=$ $\{1 / 4,-1 / 4-\omega / 4,1 / 2+\omega / 4,1 / 4-\omega / 4,-\omega / 4\}$ with code 11211 and code sum equal to six, whilst $1 / 4+\omega / 4$ 
is preperiodic to the periodic orbit $P^{2}=\{3 / 4,1 / 2-\omega / 4,1 / 4-\omega / 2,3 / 4+\omega / 4\}$ with code 2112 and code sum also equal to six. This implies that the discontinuity locations eventually have period a divisor of six. In fact the functions $Q_{n}$ have discontinuities on the fundamental interval as follows, where in the final column we note the value of $Q_{n}(0)$ for reference.

\begin{tabular}{|l|c|c|c|}
\hline$Q_{0}$ & - & - & +1 \\
\hline$Q_{1}$ & $1 / 4+\omega / 4$ & $-1 / 4-\omega / 4$ & +1 \\
\hline$Q_{2}$ & - & $1 / 2+\omega / 4$ & +1 \\
\hline$Q_{3}$ & $-1 / 4-\omega / 2$ & - & -1 \\
\hline$Q_{4}$ & $3 / 4+\omega / 4$ & $1 / 4-\omega / 4$ & -1 \\
\hline$Q_{5}$ & - & $-\omega / 4$ & +1 \\
\hline$Q_{6}$ & $3 / 4$ & $1 / 4$ & +1 \\
\hline$Q_{7}$ & - & $-1 / 4-\omega / 4$ & +1 \\
\hline$Q_{8}$ & $1 / 2-\omega / 4$ & $1 / 2+\omega / 4$ & -1 \\
\hline$Q_{9}$ & $-1 / 4-\omega / 2$ & - & -1 \\
\hline$Q_{10}$ & $3 / 4+\omega / 4$ & $1 / 4-\omega / 4$ & -1 \\
\hline
\end{tabular}

Notice the periodicity with period six from $Q_{3}$ onwards. To apply the results of the previous section we need initial conditions on the fundamental interval that are themselves periodic, and so we take for $Q_{0}$ the function with discontinuities at $1 / 4$ and $3 / 4$ only and value +1 at 0 , and for $Q_{1}$ the function with a discontinuities at $-1 / 4-\omega / 4$ only and value +1 at 0 . This initial data suffices to determine the globally periodic orbit of (3.2) for the golden mean trajectory in the half-barrier. This period 6 orbit is shown in figure 6 . Note that despite its superficial similarity, this periodic orbit is different from that considered in $[5,11]$.

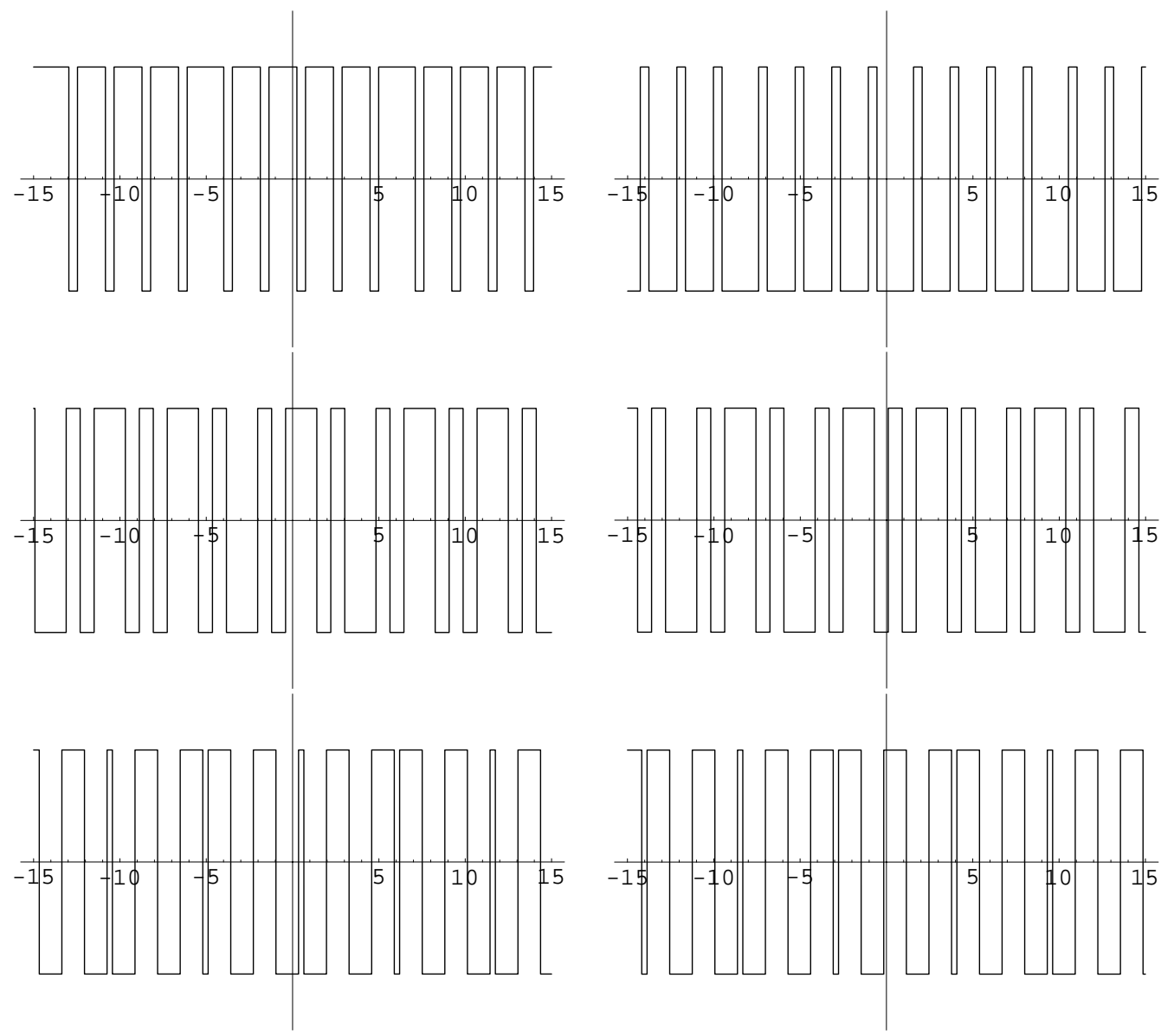

Figure 6: Period 6 orbit for the golden mean half-barrier. Reading downwards on the left $Q_{0}, Q_{1}, Q_{2}$, on the right $Q_{3}, Q_{4}, Q_{5}$. 
We remark that the discontinuities of this new $Q_{0}$ are not simply those of the barrier function $\Phi$ in the fundamental interval. Indeed $\Phi$ has period one and so has a discontinuity at $-1 / 4$ which is not a periodic point of $F$. (It is preperiodic to $P^{2}$.)

\subsection{The global discontinuity location sets}

In order to calculate the averages (2.21) we need to know the locations of the discontinuities of $Q_{n}$ on the whole of $\mathbb{R}$. In this subsection we establish results, analogous to those in [12], which give a complete description of their locations.

For $P^{1}=\{1 / 4,-1 / 4-\omega / 4,1 / 2+\omega / 4,1 / 4-\omega / 4,-\omega / 4\}$ we define the sets

$$
\begin{aligned}
& L^{1,0}=\{\lceil i \omega-1 / 4\rceil+i+1 / 4+i \omega: i \in \mathbb{Z}\}, \\
& L^{1,1}=\{\lceil(i-1 / 4) \omega\rceil+i-1 / 4+(i-1 / 4) \omega: i \in \mathbb{Z}\}, \\
& L^{1,2}=\{\lceil(i+1 / 4) \omega+3 / 4\rceil+i-1 / 2+(i+1 / 4) \omega: i \in \mathbb{Z}\}, \\
& L^{1,3}=\{\lceil(i-1 / 2) \omega+1 / 4\rceil+i-3 / 4+(i-1 / 2) \omega: i \in \mathbb{Z}\}, \\
& L^{1,4}=\{\lceil(i-1 / 4) \omega+1 / 2\rceil+i-3 / 4+(i-1 / 4) \omega: i \in \mathbb{Z}\}, \\
& L^{1,5}=\{\lceil(i-1 / 4) \omega-1 / 4\rceil+i+(i-1 / 4) \omega: i \in \mathbb{Z}\} .
\end{aligned}
$$

For $P^{2}=\{3 / 4,1 / 2-\omega / 4,1 / 4-\omega / 2,3 / 4+\omega / 4\}$ we define the sets

$$
\begin{aligned}
& L^{2,0}=\{\lceil i \omega+1 / 4\rceil+i-1 / 4+i \omega: i \in \mathbb{Z}\}, \\
& L^{2,1}=\{\lceil(i-3 / 4) \omega\rceil+i-3 / 4+(i-3 / 4) \omega: i \in \mathbb{Z}\}, \\
& L^{2,2}=\{\lceil(i-1 / 4) \omega-3 / 4\rceil+i+1 / 2+(i-1 / 4) \omega: i \in \mathbb{Z}\}, \\
& L^{2,3}=\{\lceil(i+1 / 2) \omega+3 / 4\rceil+i-1 / 4+(i+1 / 2) \omega: i \in \mathbb{Z}\}, \\
& L^{2,4}=\{\lceil(i+1 / 4) \omega+1 / 2\rceil+i-1 / 4+(i+1 / 4) \omega: i \in \mathbb{Z}\}, \\
& L^{2,5}=\{\lceil(i-3 / 4) \omega-3 / 4\rceil+i+(i-3 / 4) \omega: i \in \mathbb{Z}\} .
\end{aligned}
$$

Then we have the following.

Proposition 5. The discontinuity location sets $L_{n}$ generated by the periodic point $1 / 4$, i.e., by the periodic orbit $P^{1}$, satisfy

$$
\begin{aligned}
& L^{1,0} \cap L_{n}=\left\{\lceil i \omega-1 / 4\rceil+i+1 / 4+i \omega: i=l_{n}^{1,0}, \ldots, r_{n}^{1,0}\right\}, \\
& L^{1,1} \cap L_{n}=\left\{\lceil(i-1 / 4) \omega\rceil+i-1 / 4+(i-1 / 4) \omega: i=l_{n}^{1,1}, \ldots, r_{n}^{1,1}\right\}, \\
& L^{1,2} \cap L_{n}=\left\{\lceil(i+1 / 4) \omega+3 / 4\rceil+i-1 / 2+(i+1 / 4) \omega: i=l_{n}^{1,2}, \ldots, r_{n}^{1,2}\right\}, \\
& L^{1,3} \cap L_{n}=\left\{\lceil(i-1 / 2) \omega+1 / 4\rceil+i-3 / 4+(i-1 / 2) \omega: i=l_{n}^{1,3}, \ldots, r_{n}^{1,3}\right\} \text {, } \\
& L^{1,4} \cap L_{n}=\left\{\lceil(i-1 / 4) \omega+1 / 2\rceil+i-3 / 4+(i-1 / 4) \omega: i=l_{n}^{1,4}, \ldots, r_{n}^{1,4}\right\} \text {, } \\
& L^{1,5} \cap L_{n}=\left\{\lceil(i-1 / 4) \omega-1 / 4\rceil+i+(i-1 / 4) \omega: i=l_{n}^{1,5}, \ldots, r_{n}^{1,5}\right\},
\end{aligned}
$$

whilst those generated by $3 / 4$, i.e., by $P^{2}$, satisfy

$$
\begin{aligned}
& L^{2,0} \cap L_{n}=\left\{\lceil i \omega+1 / 4\rceil+i-1 / 4+i \omega: i=l_{n}^{2,0}, \ldots, r_{n}^{2,0}\right\}, \\
& L^{2,1} \cap L_{n}=\left\{\lceil(i-3 / 4) \omega\rceil+i-3 / 4+(i-3 / 4) \omega: i=l_{n}^{2,1}, \ldots, r_{n}^{2,1}\right\}, \\
& L^{2,2} \cap L_{n}=\left\{\lceil(i-1 / 4) \omega-3 / 4\rceil+i+1 / 2+(i-1 / 4) \omega: i=l_{n}^{2,2}, \ldots, r_{n}^{2,2}\right\}, \\
& L^{2,3} \cap L_{n}=\left\{\lceil(i+1 / 2) \omega+3 / 4\rceil+i-1 / 4+(i+1 / 2) \omega: i=l_{n}^{2,3}, \ldots, r_{n}^{2,3}\right\}, \\
& L^{2,4} \cap L_{n}=\left\{\lceil(i+1 / 4) \omega+1 / 2\rceil+i-1 / 4+(i+1 / 4) \omega: i=l_{n}^{2,4}, \ldots, r_{n}^{2,4}\right\}, \\
& L^{2,5} \cap L_{n}=\left\{\lceil(i-3 / 4) \omega-3 / 4\rceil+i+(i-3 / 4) \omega: i=l_{n}^{2,5}, \ldots, r_{n}^{2,5}\right\},
\end{aligned}
$$

$$
\begin{array}{ll}
n \equiv 0 & \bmod 6 ; \\
n \equiv 1 & \bmod 6 ; \\
n \equiv 2 & \bmod 6 ; \\
n \equiv 3 & \bmod 6 ; \\
n \equiv 4 & \bmod 6 ; \\
n \equiv 5 & \bmod 6,
\end{array}
$$

where, as $n \rightarrow \infty, l_{n}^{a, k} \rightarrow-\infty, r_{n}^{a, k} \rightarrow \infty$ for $a=1,2, k=0,1,2,3,4,5$. 
The meaning of this proposition is that, for $k=0,1,2,3,4,5$, the set $L^{1, k}$ is the limit of the sequence $\left(L_{6 m+k}\right)$ generated by $P^{1}$, with a corresponding statement for $P^{2}$.

The following lemma will be useful in the proof.

Lemma 2. Let $\eta \in \mathbb{R} \backslash \mathbb{Z}$ and let $\left(W_{n}\right)$ be a monotonic increasing sequence satisfying $W_{n} \rightarrow 1$. Define $T_{n}=\left\{i \in \mathbb{Z}:\{i \omega+\eta\} \leq W_{n}\right\}$. Then, for sufficiently large $n, T_{n}$ contains a set $\left\{l_{n}, l_{n}+1, \ldots, 0, \ldots, r_{n}-1, r_{n}\right\}$ with $l_{n} \in\{0,-1,-2, \ldots\}, r_{n} \in\{0,1,2, \ldots\}$ and $r_{n} \rightarrow \infty, l_{n} \rightarrow-\infty$ as $n \rightarrow \infty$.

Proof. Let $n_{0}=\min \left\{n \in \mathbb{N}: W_{n}>\{\eta\}\right\}$ so that $0 \in T_{n}$ for all $n \geq n_{0}$. Define $M_{n}=T_{n}^{c}=\{i \in$ $\left.\mathbb{Z}:\{i \omega+\eta\}>W_{n}\right\}$ to be the complement of $T_{n}$ and, for $n \geq n_{0}$, put $r_{n}=\min \left\{i>0: i \in M_{n}\right\}-1$, $l_{n}=\max \left\{i<0: i \in M_{n}\right\}+1$. (These numbers are well defined since $\omega$ is irrational.) Now, since $W_{n+1} \geq W_{n}$, we have $M_{n+1} \subseteq M_{n}$, and thus $T_{n} \subseteq T_{n+1}$, so that $l_{n}$ cannot increase and $r_{n}$ cannot decrease. It remains to prove that the sequences $\left(l_{n}\right)$ and $\left(r_{n}\right)$ are unbounded. Suppose that $\left(r_{n}\right)$ is bounded above. Then there exists $\hat{n} \in \mathbb{N}$ such that $r_{n}=r_{\hat{n}}$ for all $n \geq \hat{n}$. Then $\{\hat{n} \omega+\eta\}>W_{n}$ for all $n \geq \hat{n}$. However $\{\hat{n} \omega+\eta\}$ is fixed, so we have a contradiction with $W_{n} \rightarrow 1$. Thus $r_{n} \rightarrow+\infty$. A similar argument shows that $l_{n} \rightarrow-\infty$.

Proof of Proposition 5. The following easily verified table for the Fibonacci numbers modulo 4 will be useful.

\begin{tabular}{|c|c|}
\hline$n \bmod 6$ & $F_{n} \bmod 4$ \\
\hline 0 & 0 \\
\hline 1 & 1 \\
\hline 2 & 1 \\
\hline 3 & 2 \\
\hline 4 & 3 \\
\hline 5 & 1 \\
\hline
\end{tabular}

(a) We firstly consider the discontinuity location sets $L_{n}$ generated by the periodic orbit $P^{1}$, for which the discontinuity of $Q_{0}$ is at $1 / 4$. We shall only prove the result in the cases $n \equiv 0$ and $n \equiv 1 \bmod 6$, the remaining cases being similar.

(i) $n \equiv 0 \bmod 6$

According to Proposition 4, $n$ being even, we have

$$
L_{n}=\left\{F_{n+1} / 4+\lceil i \omega\rceil+i+\left(i+F_{n} / 4\right) \omega: i=-F_{n}, \ldots, F_{n-1}-1\right\} .
$$

Replacing $i$ by $i-F_{n} / 4$ (noting that $F_{n} / 4,\left(F_{n+1}-1\right) / 4 \in \mathbb{Z}$ ) we see that

$$
\begin{aligned}
L_{n} & =\left\{F_{n+1} / 4+\left\lceil\left(i-F_{n} / 4\right) \omega\right\rceil+i-F_{n} / 4+i \omega: i=-3 F_{n} / 4, \ldots, F_{n} / 4+F_{n-1}-1\right\} \\
& =\left\{\left\lceil i \omega+\left(F_{n+1}-F_{n}-1-F_{n} \omega\right) / 4\right\rceil+i+1 / 4+i \omega: i=-3 F_{n} / 4, \ldots, F_{n} / 4+F_{n-1}-1\right\} .
\end{aligned}
$$

Now, using $F_{n-1}-F_{n} \omega=\omega^{n}, n$ being even,

$$
\left\lceil i \omega+\left(F_{n+1}-F_{n}-1-F_{n} \omega\right) / 4\right\rceil=\left\lceil i \omega-1 / 4+\omega^{n} / 4\right\rceil,
$$

so we are required to show that

$$
\left\lceil i \omega-1 / 4+\omega^{n} / 4\right\rceil=\lceil i \omega-1 / 4\rceil,
$$

for a suitable range of $i$. Now (4.15) is true if, and only if, $\lceil i \omega-1 / 4\rceil \geq i \omega-1 / 4+\omega^{n} / 4$, i.e., if, and only if,

$$
i \omega-\lceil i \omega-1 / 4\rceil+3 / 4 \leq 1-\omega^{n} / 4 .
$$

But

$$
i \omega-\lceil i \omega-1 / 4\rceil+3 / 4=i \omega-1 / 4-\lceil i \omega-1 / 4\rceil+1=\{i \omega-1 / 4\},
$$

using the fact that $\{x\}=1-(\lceil x\rceil-x)$ for all $x \in \mathbb{R} \backslash \mathbb{Z}$, and thus (4.16) has a form suitable for application of Lemma 2. We thus deduce that (4.15) is true for a suitable range of $i$ as required. 
(ii) $n \equiv 1 \bmod 6$

In this case $n$ is odd and Proposition 4 gives

$$
L_{n}=\left\{-F_{n+1} / 4+\lceil i \omega\rceil+i+\left(i-F_{n} / 4\right) \omega: i=-F_{n-1}, \ldots, F_{n}-1\right\},
$$

which, replacing $i$ by $i+\left(F_{n}-1\right) / 4$ and proceeding as above (noting that $\left.\left(F_{n}-1\right) / 4,\left(F_{n+1}-1\right) / 4 \in \mathbb{Z}\right)$ gives

$$
L_{n}=\left\{\left\lceil(i-1 / 4) \omega+\omega^{n} / 4\right\rceil+i-1 / 4+(i-1 / 4) \omega: i=-F_{n-1}-\left(F_{n}-1\right) / 4, \ldots, 3\left(F_{n}-1\right) / 4\right\} .
$$

We are thus required to show that

$$
\left\lceil(i-1 / 4) \omega+\omega^{n} / 4\right\rceil=\lceil(i-1 / 4) \omega\rceil,
$$

for a suitable range of $i$. Now (4.17) is true if, and only if, $\lceil(i-1 / 4) \omega\rceil \geq(i-1 / 4) \omega+\omega^{n} / 4$, i.e., if, and only if, $(i-1 / 4) \omega-\lceil(i-1 / 4) \omega\rceil+1 \leq 1-\omega^{n} / 4$, but, as above, this is just

$$
\{(i-1 / 4) \omega\} \leq 1-\omega^{n} / 4,
$$

and so we may again use Lemma 2 to deduce the desired result.

(b) For the discontinuity location sets $L_{n}$ generated by the periodic orbit $P^{2}$, for which the discontinuity of $Q_{0}$ is at $3 / 4$, we shall only prove the result in the case $n \equiv 0 \bmod 6$. In this case, $n$ being even, Proposition 4 gives

$$
L_{n}=\left\{3 F_{n+1} / 4+\lceil i \omega\rceil+i+\left(i+3 F_{n} / 4\right) \omega: i=-F_{n}, \ldots, F_{n-1}-1\right\},
$$

which on replacing $i$ by $i-3 F_{n} / 4$ and proceeding as above (noting $F_{n} / 4,\left(F_{n+1}-1\right) / 4 \in \mathbb{Z}$ ) gives

$$
L_{n}=\left\{\left\lceil i \omega+1 / 4+3 \omega^{n} / 4\right\rceil+i-1 / 4+i \omega: i=-F_{n} / 4, \ldots, 3 F_{n} / 4+F_{n-1}-1\right\},
$$

so that we are required to show that

$$
\left\lceil i \omega+1 / 4+3 \omega^{n} / 4\right\rceil=\lceil i \omega+1 / 4\rceil
$$

for a suitable range of $i$. Now (4.19) is true if, and only if, $\lceil i \omega+1 / 4\rceil \geq i \omega+1 / 4+3 \omega^{n} / 4$. However, as in (a) above, this is the statement that

$$
\{i \omega+1 / 4\} \leq 1-3 \omega^{n} / 4,
$$

and so we may again use Lemma 2 to deduce the desired result.

\subsection{Combining the discontinuities}

Having identified the locations of the two sets of discontinuities we now see how they combine. This is needed in order to be able to calculate the averages (2.21), which we shall do in the following subsection.

We denote $L_{i}^{a, n}$ to be the $i^{\text {th }}$ member of the set $L^{a, n}, a=1,2$. We firstly note from Proposition 4 that, for $a=1,2$,

$$
L_{i+1}^{a, n}-L_{i}^{a, n}=(1 \text { or } 2)+\omega .
$$

We now investigate how these two sets of discontinuities interlace.

4.3.1 $n \equiv 0(\bmod 3)$

When $n \equiv 0(\bmod 6)$, from (4.3) and (4.9), we have

$$
\begin{aligned}
L_{i}^{2,0}-L_{i}^{1,0} & =(\lceil i \omega+1 / 4\rceil+i-1 / 4+i \omega)-(\lceil i \omega-1 / 4\rceil+i+1 / 4+i \omega) \\
& =-1 / 2+\lceil i \omega+1 / 4\rceil-\lceil i \omega-1 / 4\rceil \\
& =-1 / 2+(0 \text { or } 1)= \pm 1 / 2 .
\end{aligned}
$$


If $L_{i}^{2,0}-L_{i}^{1,0}=+1 / 2$, using (4.21), we then have possible configurations as shown in figure 7 , with similar configurations when $L_{i}^{2,0}-L_{i}^{1,0}=-1 / 2$. We cannot have two adjacent intervals of length $1 / 2$, for then either $L_{i+1}^{1,0}-L_{i}^{1,0}$ or $L_{i+1}^{2,0}-L_{i}^{2,0}=1$ which contradicts (4.21). Because the initial condition $Q_{0}$, with its discontinuities at $1 / 4$ and $3 / 4$ and value +1 at zero, contains such an interval of length $1 / 2$ in which the value is -1 , on all of the intervals of length $1 / 2$ the value is -1 . (See figure 6 top left.)
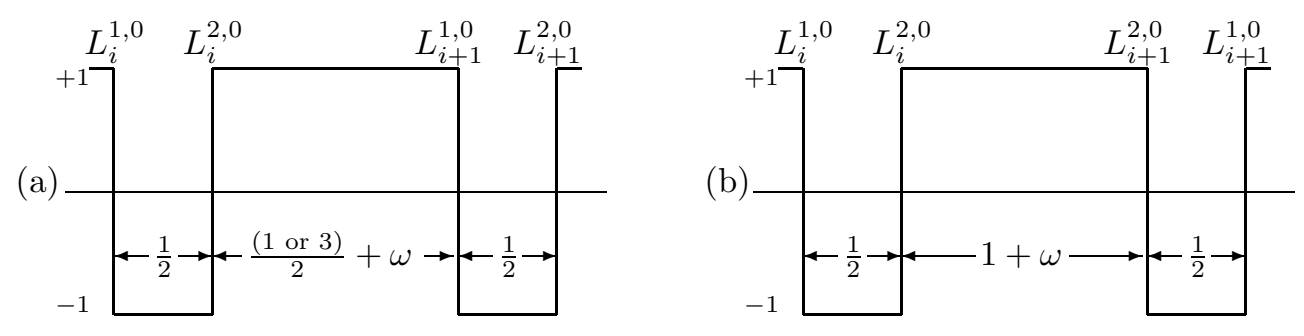

Figure 7: Allowed configurations for the interlacing of $L^{1,0}$ and $L^{2,0}$

The graph of $Q_{n}$ thus consists of intervals of length $1 / 2$ with value -1 punctuated by intervals of length $(1 / 2$ or 1 or $3 / 2)+\omega$ with value +1 . From these considerations alone we can see that the autocorrelation $C\left(F_{n}\right)$ will take a value strictly between 0 and 1 for these values of $n$. We shall give a precise evaluation in the next subsection.

When $n \equiv 3(\bmod 6)$, from $(4.6)$ and $(4.12)$, we have

$$
\begin{aligned}
L_{i+1}^{1,3}-L_{i}^{2,3} & =(\lceil(i+1 / 2) \omega+1 / 4\rceil+i+1 / 4+(i+1 / 2) \omega)-(\lceil(i+1 / 2) \omega+3 / 4\rceil+i-1 / 4+(i+1 / 2) \omega) \\
& =1 / 2+\lceil(i+1 / 2) \omega+1 / 4\rceil-\lceil(i+1 / 2) \omega+3 / 4\rceil \\
& =1 / 2+(0 \text { or }-1)= \pm 1 / 2
\end{aligned}
$$

so that the allowed configurations are as for the case $n \equiv 0(\bmod 6)$, except that the graphs of figure 7 are multiplied by -1 , so that the excursions of length $1 / 2$ now have positive sign. (See figure 6 top right.) As a consequence the autocorrelation now lies strictly between -1 and 0 .

4.3.2 $n \equiv 1(\bmod 3)$

For $n \equiv 1(\bmod 6)$, from $(4.4)$ and $(4.10)$, we have

$$
\begin{aligned}
L_{i}^{1,1}-L_{i}^{2,1} & =\lceil(i-1 / 4) \omega\rceil+i-1 / 4+(i-1 / 4) \omega-(\lceil(i-3 / 4) \omega\rceil+i-3 / 4+(i-3 / 4) \omega) \\
& =1 / 2+\omega / 2+\lceil(i-1 / 4) \omega\rceil-\lceil(i-3 / 4) \omega\rceil \\
& =(1 \text { or } 3) / 2+\omega / 2>0,
\end{aligned}
$$

and

$$
\begin{aligned}
L_{i+1}^{2,1}-L_{i}^{1,1} & =\lceil(i+1 / 4) \omega\rceil+i+1 / 4+(i+1 / 4) \omega-(\lceil(i-1 / 4) \omega\rceil+i-1 / 4+(i-1 / 4) \omega) \\
& =1 / 2+\omega / 2+\lceil(i+1 / 4) \omega\rceil-\lceil(i-1 / 4) \omega\rceil \\
& =(1 \text { or } 3) / 2+\omega / 2>0 .
\end{aligned}
$$

Thus the two sets of discontinuities are strictly alternating: $\cdots<L_{i}^{2,1}<L_{i}^{1,1}<L_{i+1}^{2,1}<L_{i+1}^{1,1}<\cdots$.

We now partition $\mathbb{R}$ into the intervals $\left[L_{i}^{1,1}, L_{i+1}^{1,1}\right]$ and see, using (4.21), that the allowed configurations are as shown in figure 8. Note that, by the initial conditions $L_{0}^{1,1}=-1 / 4-\omega / 4$ and $Q_{1}(0)=+1$, for these $n$ the value of $Q_{n}$ is +1 from $L_{i}^{1,1}$ to $L_{i+1}^{2,1}$ and -1 from $L_{i+1}^{2,1}$ to $L_{i+1}^{1,1}$ as shown.

We shall show below that the number of occurrences of figures $8(a)$ and (b) are equal so that the integral (2.21) is zero in this case. Note that all the intervals of length $1+\omega$ as shown figure $8(\mathrm{c})$ will make no contribution to the integral.

When $n \equiv 4(\bmod 6)$ we have the same possible configurations as for $n \equiv 1(\bmod 6)$ except that the ordering is now $\cdots<L_{i}^{2,4}<L_{i+1}^{1,4}<L_{i+1}^{2,4}<L_{i+2}^{1,4}<\cdots$. 

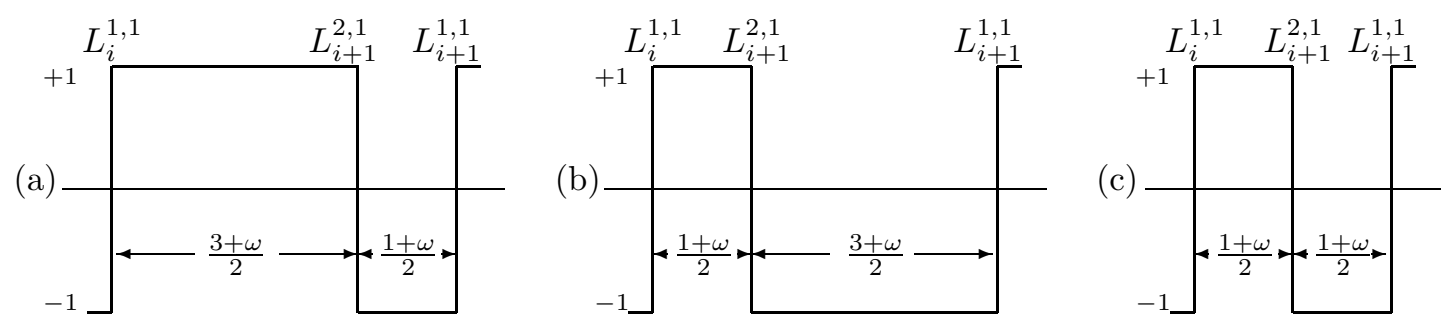

Figure 8: Possibilities for each interval between $L_{i}^{1,1}$ and $L_{i+1}^{1,1}$

4.3.3 $n \equiv 2(\bmod 3)$

For $n \equiv 2(\bmod 6)$, from $(4.5)$ and $(4.11)$, we have

$$
\begin{aligned}
L_{i}^{1,2}-L_{i}^{2,2} & =\lceil(i+1 / 4) \omega+3 / 4\rceil+i-1 / 2+(i+1 / 4) \omega-(\lceil(i-1 / 4) \omega-3 / 4\rceil+i+1 / 2+(i-1 / 4) \omega) \\
& =-1+\omega / 2+\lceil(i+1 / 4) \omega+3 / 4\rceil-\lceil(i-1 / 4) \omega-3 / 4\rceil \\
& =-1+\omega / 2+(1 \text { or } 2)=(0 \text { or } 1)+\omega / 2>0
\end{aligned}
$$

and

$$
\begin{aligned}
L_{i+1}^{2,2}-L_{i}^{1,2} & =\lceil(i+3 / 4) \omega-3 / 4\rceil+i+3 / 2+(i+3 / 4) \omega-(\lceil(i+1 / 4) \omega+3 / 4\rceil+i-1 / 2+(i+1 / 4) \omega) \\
& =1+\omega / 2+\lceil(i+3 / 4) \omega-3 / 4\rceil-\lceil(i+1 / 4) \omega+3 / 4\rceil \\
& =1+\omega / 2+(-1 \text { or } 0)=(0 \text { or } 1)+\omega / 2>0
\end{aligned}
$$

so, as in the case $n \equiv 1(\bmod 6)$, we have $\cdots<L_{i}^{2,2}<L_{i}^{1,2}<L_{i+1}^{2,2}<L_{i+1}^{1,2}<\cdots$. Partitioning $\mathbb{R}$ into the intervals $\left[L_{i}^{1,2}, L_{i+1}^{1,2}\right]$, using (4.21), the allowable configurations are now as in figure 9 . Note that, by the initial conditions $L_{0}^{2,2}=1 / 2-\omega / 4$ and $Q_{2}(0)=-1$, for these $n$ the value of $Q_{n}$ is -1 from $L_{i}^{1,2}$ to $L_{i+1}^{2,2}$ and +1 from $L_{i+1}^{2,2}$ to $L_{i+1}^{1,2}$ as shown.
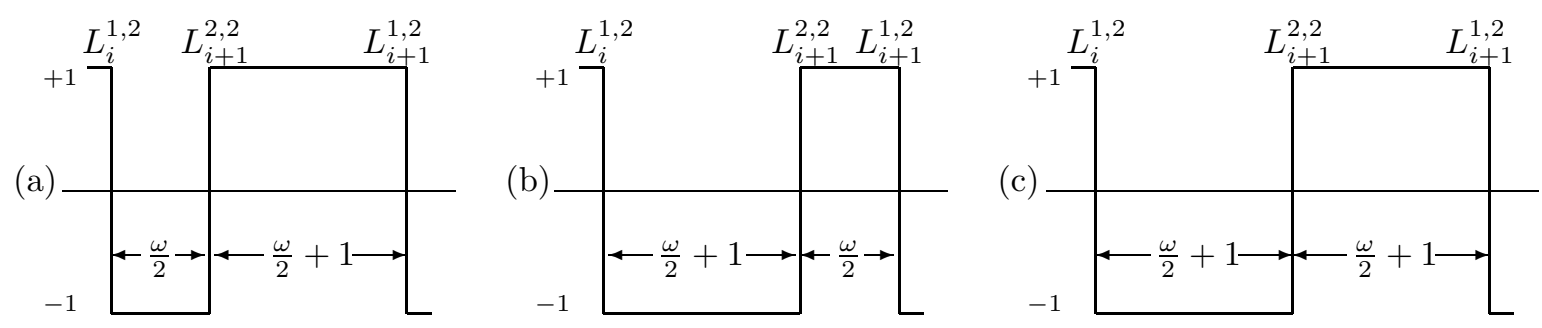

Figure 9: Possibilities for each interval between $L_{i}^{1,2}$ and $L_{i+1}^{1,2}$

We shall show below that the number of occurrences of figures $9(\mathrm{a})$ and (b) are equal so that the integral (2.21) is also zero in this case. Note that the intervals of length $2+\omega$ as shown figure 9 (c) make no contribution to the integral.

When $n \equiv 5(\bmod 6)$ we have the same possible configurations as for $n \equiv 2(\bmod 6)$ with exception of the ordering. We now have $\cdots<L_{i}^{2,5}<L_{i}^{1,5}<L_{i+1}^{2,5}<L_{i+1}^{1,5}<\cdots$.

\subsection{Evaluation of $C\left(F_{n}\right)$}

4.4.1 $n \equiv 0(\bmod 3)$

Theorem 1. For the golden mean trajectory of the half-barrier we have

$$
\lim _{m \rightarrow \infty} C\left(F_{6 m+3 k}\right)=(-1)^{k}(1-1 / \sqrt{5}) .
$$


Proof. We must calculate the limit of the integral (2.21). The calculation is similar to that in [12].

When $n \equiv 0(\bmod 6)$, i.e., $k=0$, as we saw in Subsection 4.3 .1 (see figure 7 and figure 6 top left), the graph of $Q_{n}$ consists of intervals of length $1 / 2$ on which the value is -1 , the value, of course, being +1 elsewhere. Each of these intervals has associated with it a discontinuity from $L^{1,0}$. On an interval of length $\omega^{-n}=F_{n+1}+F_{n} \omega+O(1)$ there are $F_{n}+O(1)$ such points. Thus

$$
C\left(F_{n}\right)=\frac{1}{(-\omega)^{-n}} \int_{0}^{(-\omega)^{-n}} Q_{n}(x) d x=\frac{1}{\omega^{-n}}\left(\omega^{-n}-2 \times \frac{1}{2}\left(F_{n}+O(1)\right)\right) \rightarrow 1-\frac{1}{\sqrt{5}} .
$$

When $n \equiv 3(\bmod 6)$, the calculation is similar.

4.4.2 $n \equiv 1,2(\bmod 3)$

We now show that the autocorrelation function is asymptotically 0 at all other Fibonacci numbers. We remark that the corresponding result for the quasiperiodically forced two-level quantum system [12] was immediate, but requires further detailed analysis of the allowable configurations of the interlacing discontinuities here.

Theorem 2. For the golden mean trajectory of the half-barrier we have

$$
\lim _{m \rightarrow \infty} C\left(F_{3 m+k}\right)=0, \quad k=1,2 .
$$

Proof. (a) $k=1$

We shall only give details for the case $n \equiv 1(\bmod 6)$ since the case $n \equiv 4(\bmod 6)$ is similar.

From Subsection 4.3.2 we know that the only contribution to the integral (2.21) is that from a $2+\omega$ interval, so suppose that $L_{i+1}^{1,1}-L_{i}^{1,1}=2+\omega$. Now, by (4.4) we have

$$
L_{i+1}^{1,1}-L_{i}^{1,1}=\lceil(i+3 / 4) \omega\rceil-\lceil(i-1 / 4) \omega\rceil+1+\omega,
$$

and so a necessary and sufficient condition for $\left[L_{i}^{1,1}, L_{i+1}^{1,1}\right]$ to be a $2+\omega$ interval is

$$
\lceil(i+3 / 4) \omega\rceil-\lceil(i-1 / 4) \omega\rceil=1 .
$$

For convenience, we set $r=\lceil(i-1 / 4) \omega\rceil$. Then we can rewrite (4.31) as $r-\omega<(i-1 / 4) \omega<r$, i.e.,

$$
r-3 \omega / 4<i \omega<r+\omega / 4
$$

so that $i \omega$ lies in an interval (of length less than one) with centre $r-\omega / 4$.

Now let $2^{+}$denote the $2+\omega$ interval shown in figure 8 (a) and let $2^{-}$denote the $2+\omega$ interval shown in figure 8 (b). In other words, if $L_{i+1}^{2,1}-L_{i}^{1,1}=3 / 2+\omega / 2$ we say that we have a $2^{+}$interval, whilst if $L_{i+1}^{2,1}-L_{i}^{1,1}=1 / 2+\omega / 2$ we say that we have a $2^{-}$interval. From (4.4) and (4.10) we have

$$
L_{i+1}^{2,1}-L_{i}^{1,1}=\lceil(i+1 / 4) \omega\rceil-\lceil(i-1 / 4) \omega\rceil+1 / 2+\omega / 2,
$$

thus we have $2^{+}$interval whenever

$$
\lceil(i+1 / 4) \omega\rceil-\lceil(i-1 / 4) \omega\rceil=1,
$$

and a $2^{-}$interval whenever

$$
\lceil(i+1 / 4) \omega\rceil-\lceil(i-1 / 4) \omega\rceil=0 .
$$

Since the integral over a $2^{+}$interval is minus that over a $2^{-}$interval, in order to show the total integral is zero (all other contributions being zero) it suffices to show that there are an equal number of $2^{-}$and $2^{+}$ intervals.

Now (4.33-4.34) show that we have a $2^{+}$interval whenever

$$
i \omega<r-\omega / 4
$$


and a $2^{-}$interval whenever

$$
i \omega>r-\omega / 4
$$

Thus, by (4.32), we see that the intervals (in which $i \omega$ lies) corresponding to $2^{+}$and $2^{-}$have equal length. Hence by the uniform distribution of $\{i \omega\}$ we have the same number of $2^{+}$'s and $2^{-}$'s, and hence the (asymptotic) value of the integral is zero.

(b) $k=2$

We shall only give the details for the case $n \equiv 2(\bmod 6)$, omitting the case $n \equiv 5(\bmod 6)$, which is similar. In Subsection 4.3.3 we saw that it is only the $1+\omega$ intervals between $L_{i}^{1,2}$ and $L_{i+1}^{1,2}$ that contribute to the integral, so suppose that $L_{i+1}^{1,2}-L_{i}^{1,2}=1+\omega$. By (4.6) we have

$$
L_{i+1}^{1,2}-L_{i}^{1,2}=\lceil(i+5 / 4) \omega+3 / 4\rceil-\lceil(i+1 / 4) \omega+3 / 4\rceil+1+\omega
$$

and so a necessary and sufficient condition for a $1+\omega$ interval is

$$
\lceil(i+5 / 4) \omega+3 / 4\rceil-\lceil(i+1 / 4) \omega+3 / 4\rceil=0
$$

We set $r=\lceil(i+1 / 4) \omega+3 / 4\rceil$ and thus rewrite $(4.37)$ as $r-1<(i+1 / 4) \omega+3 / 4<r-\omega$, i.e.,

$$
r-7 / 4-\omega / 4<i \omega<r-3 / 4-5 \omega / 4
$$

so that $i \omega$ lies in an interval (of length less than one) with centre $r-5 / 4-3 \omega / 4$.

We now let $1^{+}$denote $1+\omega$ interval shown in figure $9(\mathrm{a})$ and let $1^{-}$denote the $1+\omega$ interval shown in figure 9(b). That is if $L_{i+1}^{2,2}-L_{i}^{1,2}=\omega / 2$ we say that we have a $1^{+}$interval, whilst if $L_{i+1}^{2,2}-L_{i}^{1,2}=1+\omega / 2$ we say that we have a $1^{-}$interval. From (4.5) and (4.11) we have

$$
L_{i+1}^{2,2}-L_{i}^{1,2}=\lceil(i+3 / 4) \omega+1 / 4\rceil-\lceil(i+1 / 4) \omega+3 / 4\rceil+1+\omega / 2,
$$

thus we have $1^{+}$interval whenever

$$
\lceil(i+3 / 4) \omega+1 / 4\rceil-\lceil(i+1 / 4) \omega+3 / 4\rceil=-1
$$

and a $1^{-}$interval whenever

$$
\lceil(i+3 / 4) \omega+1 / 4\rceil-\lceil(i+1 / 4) \omega+3 / 4\rceil=0 .
$$

As in the case $k=1$ it is enough to show that there are an equal number of $1^{-}$and $1^{+}$intervals.

So suppose that we have a $1+\omega$ interval from $L_{i}^{1,2}$ to $L_{i+1}^{1,2}$, so that (4.38) holds. Then (4.39-4.40) show that we have a $1^{+}$interval whenever

$$
i \omega>r-3 \omega / 4-5 / 4
$$

and a $1^{-}$interval whenever

$$
i \omega<r-3 \omega / 4-5 / 4
$$

Thus, by (4.38), we see that the intervals (in which $i \omega$ lies) corresponding to $1^{+}$and $1^{-}$have equal length. Hence by the uniform distribution of $\{i \omega\}$ we have the same number of $1^{+}$'s and $1^{-}$'s, and hence the (asymptotic) value of the integral is again zero.

\section{Conclusion}

We have given a rigorous renormalization analysis of the self-similarity of autocorrelation functions in symmetric barrier billiards for golden mean trajectories. Key to this analysis is the functional recurrence (1.1), the initial conditions to which are dictated by the barrier geometry. The analysis draws heavily on our recent work of fluctuations in the Harper equation [14], and of correlations in strange non-chaotic attractors [11] 
and in quantum two-level systems[12]. For the special case of the half-barrier we gave a rigorous calculation of the asymptotic values in the autocorrelation function shown in figure 3 at Fibonacci numbers. The main peaks occur at every third Fibonacci number and have asymptotic height $1-1 / \sqrt{5} \approx 0.55$, with value zero at other Fibonacci numbers.

As mentioned in Section 4, a barrier with an arbitrary finite number of pieces may be decomposed into single barriers. If this single barrier has endpoint in $\mathbb{Q}(\omega)$ then there will be exact asymptotic self-similarity of the autocorrelation function, corresponding to periodicity under (1.1), as in the half-barrier case treated in detail here. Generally however we expect there to be a renormalization strange set on which the renormalization dynamics takes place. Such a set - the orchid - arises in a generalised Harper equation [8], which we have recently treated rigorously in [13]. Dynamics under (1.1) would then be typically chaotic, and we might say that we have "chaotic correlations." An important ingredient is the presence of a symmetry in the initial conditions to (1.1), which manifests itself as a symmetry in the renormalization strange set. We shall have more to say about arbitrary barriers in a future article.

The results in this article are limited to only one trajectory, namely the golden mean. It will be straightforward to generalise to quadratic irrationals, as has been done in [2] for the renormalization fixed point associated with the Harper equation. An appropriate generalisation of recurrence (1.1) governs the behaviour. The generalisation to arbitrary irrational numbers should also be possible. In this case a renormalization scheme in which the recurrence changes from step-to-step is appropriate. We intend to treat this problem in future works.

\section{Acknowledgement}

The authors would like to acknowledge valuable discussions with Ben Mestel.

\section{References}

[1] A. Bondeson, E. Ott, and T. M. Antonsen, Quasiperiodically forced damped pendula and Schrödinger equations with quasiperiodic potentials: implications of their equivalence, Phys. Rev. B 55 (1985), 21032106.

[2] J. Dalton and B. D. Mestel, Renormalisation for the Harper equation for quadratic irrationals, preprint (2002).

[3] B. Eckhardt, J. Ford, and F. Vivaldi, Analytically solvable dynamical systems which are not integrable, Physica D 13 (1984), 339-356.

[4] A. Eskin, H. Masur, and M. Schmoll, Billiards in rectangles with barriers, preprint math.DS/0107204 (2001).

[5] U. Feudel, A. Pikovsky, and A. Politi, Renormalization of correlations and spectra of a strange nonchaotic attractor, J. Phys. A 29 (1996), 5297-5311.

[6] U. Feudel, A.S. Pikovsky, and M. A. Zaks, Correlation properties of a quasiperiodically forced two-level system, Phys. Rev. E 51 (1995), 1762-1769.

[7] J. H. Hannay and R. J. McCraw, Barrier billiards-a simple pseudo-integrable system, J. Phys. A 23 (1990), 887-900.

[8] J. A. Ketoja and I. I. Satija, Self-similarity and localization, Phys. Rev. Lett. 75 (1995), 2762-2765.

[9] J. A. Ketoja and I. I. Satija, Harper equation, the dissipative standard map and strange nonchaotic attractors: Relationship between an eigenvalue problem and iterated maps, Physica D 109 (1997), 7080. 
[10] S. P. Kuznetsov, A. S. Pikovsky, and U. Feudel, Birth of a strange nonchaotic attractor: A renormalization group analysis, Phys. Rev. E 51 (1995), R1629-R1632.

[11] B. D. Mestel and A. H. Osbaldestin, Periodic orbits of renormalisation for the correlations of strange nonchaotic attractors, Math. Phys. EJ 6 (2000), no. 5, 27 pp.

[12] B. D. Mestel and A. H. Osbaldestin, Renormalization analysis of correlation properties in a quasiperiodically forced two-level system, J. Math. Phys. 43 (2002), 3458-3483.

[13] B. D. Mestel and A. H. Osbaldestin, Golden mean renormalisation for a generalised Harper equation: the Ketoja-Satija orchid, preprint (2002).

[14] B. D. Mestel, A. H. Osbaldestin, and B. Winn, Golden mean renormalization for the Harper equation: the strong coupling fixed point, J. Math. Phys. 41 (2000), 8304-8330.

[15] A. S. Pikovsky and U. Feudel, Correlations and spectra of strange non-chaotic attractors, J. Phys. A 27 (1994), 5209-5219.

[16] G. W. Riley, On spectral properties of skew products over irrational rotations, J. London Math. Soc. 17 (1978), 152-160.

[17] J. Wiersig, Singular continuous spectra in a pseudointegrable billiard, Phys. Rev. E 62 (2000), R21-R24.

[18] J. Wiersig, Pseudointegrable Andreev billiard, Phys. Rev. E 65 (2002), 036221, 5 pp.

[19] G. M. Zaslavsky and M. Edelman, Weak mixing and anomalous kinetics along filamented surfaces, Chaos 11 (2001), 295-305. 